\title{
Erratum to: Effect of carbonylcyanide-4-(trifluoromethoxy) phenylhydrazone (FCCP) on the interaction of 1-anilino-8-naphthalene sulfonate (ANS) with phosphatidylcholine liposomes
}

\author{
Andrea C. Cutró • Guillermo G. Montich • \\ Oscar A. Roveri
}

Published online: 21 March 2014

(C) Springer Science+Business Media New York 2014

Erratum to: J Bioenerg Biomembr (2014)

DOI 10.1007/s10863-014-9545-0

The original version of this article has an error in Equation 4.

The correct equation is:

$\Delta F_{1}^{\infty}=\left(\Delta F_{1}^{\infty}\right)_{[F C C P]=\infty}+\frac{\left(\left(\Delta F_{1}^{\infty}\right)_{[F C C P]=0}-\left(\Delta F_{1}^{\infty}\right)_{[F C C P]=\infty}\right) \times C_{0.5}}{C_{0.5}+[F C C P]}$

The online version of the original article can be found at http://dx.doi.org/ 10.1007/s10863-014-9545-0.

A. C. Cutró · O. A. Roveri $(\bowtie)$

Area Biofísica, Facultad de Ciencias Bioquímicas y Farmacéuticas,

Universidad Nacional de Rosario, Suipacha 531,

S2002LRK Rosario, Argentina

e-mail: oroveri@fbioyf.unr.edu.ar

A. C. Cutró

e-mail: andreacutro@yahoo.com.ar

G. G. Montich

Centro de Investigaciones en Química Biológica de Córdoba

(CIQUIBIC, UNC-CONICET), Departamento de Química

Biológica, Facultad de Ciencias Químicas, Universidad Nacional de

Córdoba, Córdoba, Argentina

e-mail: gmontich@fcq.unc.edu.ar

Present Address:

A. C. Cutró

Laboratorio de Biointerfases y Sistemas Biomiméticos, Laboratorios

Centrales Centro de Investigación y Transferencia Santiago del

Estero, CITSE UNSE CONICET, El Zanjón, RN 9, km1125,

Santiago del Estero, Argentina 